\title{
LOS OJOS DEL ÁRBOL: PERCIBIENDO, REGISTRANDO, COMPRENDIENDO Y CONTRARRESTANDO LAS INVASIONES BIOLÓGICAS EN TIEMPOS DE RÁPIDA HOMOGENEIZACION BIOCULTURAL
}

\author{
RAMIRO D. CREGO',e, NORA WARD ${ }^{\mathrm{b}}$, JAIME E. JIMÉNEZ ${ }^{\mathrm{a}, \mathrm{b}, \mathrm{c}, \mathrm{d}, e}$, FRANCISCA \\ MASSARDO ${ }^{\mathrm{d}, e}$ \& RICARDO ROZZI $\mathrm{l}^{\mathrm{b}, \mathrm{d}, \mathrm{d}, \mathrm{e}}$
}

\section{RESUMEN}

El Antropoceno está marcado por un pervasivo proceso de homogeneización biocultural que conlleva pérdidas de biodiversidad y de culturas. Dicha homogeneización se evidencia en el caso de algunas invasiones biológicas. La ética biocultural procura contrarrestar tal homogeneización. Para este fin se ha diseñado una aproximación metodología denominada Filosofía Ambiental de Campo (FILAC). La FILAC se ha gestado e implementado en el sitio más austral de América de la Red Internacional de Estudios Ecológicos a Largo Plazo (ILTER), el Parque Etnobotánico Omora, con el fin de integrar estudios ecológicos con estudios filosóficos en prácticas de conservación biocultural. Los sitios ILTER tienen por objetivo investigar y monitorear el cambio socio-ambiental global. Al mismo tiempo, procuran contribuir a reorientar este cambio hacia trayectorias de sustentabilidad socio-ambiental. Sin embargo, para estos fines la red ILTER presenta tres limitaciones importantes: (i) está focalizada en estudios biofísicos, omitiendo dimensiones culturales que pueden ser relevantes, (ii) está centrada en estudios teóricos, postergando estudios aplicados, y (iii) la distribución de los sitios ILTER tiene un marcado sesgo geográfico hacia el hemisferio norte. El objetivo de este trabajo es contribuir a subsanar las tres limitaciones identificadas mediante la integración de componentes ecológicos, filosóficos y tecnológicos en base al trabajo sobre una especie invasora que ha arribado recientemente al extremo austral de América, el visón norteamericano (Neovison vison). Un resultado principal del trabajo es la composición de la metáfora "los ojos del árbol". Esta metáfora integra la investigación sobre formas de conocimiento y cosmovisiones científicas, filosóficas y amerindias y convoca acciones de conservación de modo transdisciplinario. Esta metáfora invita a una mirada más contextual para observar, registrar y comprender la problemática de las especies invasoras. Contribuye a contrarrestar el impacto de las especies exóticas mediante acciones de educación y control que priorizan el ecosistema como un todo. De esta manera, complementa enfoques valóricos que privilegian solo el bienestar de cada individuo, por sobre la integridad de la comunidad biótica.

PALABRAS CLAVE: Filosofía Ambiental de Campo, especies invasoras, homogeneización biocultural, ILTER, LTSER.

a Department of Biological Sciences, University of North Texas, Denton, TX, USA.

b Department of Philosophy and Religion, University of North Texas, Denton, TX, USA.

c Instituto de Ecología y Biodiversidad, Departamento de Ciencias Ecológicas, Facultad de Ciencias, Santiago, Chile.

$\triangle$ ramirocrego84@gmail.com

d Universidad de Magallanes, Punta Arenas, Chile.

e Sub-Antarctic Biocultural Conservation Program, University of North Texas, Denton, TX, USA. 


\title{
THE EYES OF THE TREE: PERCEIVING, MONITORING, UNDERSTANDING, AND COUNTERING BIOLOGICAL INVASIONS IN TIMES OF RAPID BIOCULTURAL HOMOGENIZATION
}

\begin{abstract}
The Anthropocene is marked by a pervasive process of biocultural homogenization that includes losses of biological and cultural diversity. The process is evident in the case of some biological invasions. Biocultural ethics aims to counteract this homogenization process. Toward this aim, the Field Environmental Philosophy (FEP) methodological approach has been designed. FEP has been conceived and implemented at the Americas' southernmost site of the International Long-term Ecological Research (ILTER) network, the Omora Ethnobotanical Park, to integrate ecological and philosophical research into biocultural conservation. ILTER sites are intended to investigate and monitor global socio-environmental change. At the same time, ILTER sites aim to contribute reorienting this change toward trajectories of socio-environmental sustainability. However, to achieve these aims the ILTER network presents three major limitations: (i) it is focused on biophysical research, largely ignoring relevant cultural dimensions; (ii) it is focused on theoretical studies, frequently postponing applied studies; (iii) and the distribution of ILTER sites has a marked geographical bias towards the northern hemisphere. The objective of this work is to contribute to overcome these three limitations by integrating ecological, philosophical, and technological components on the basis of the work conducted on an invasive species that has recently arrived to the southern end of the Americas, the American mink (Neovison vison). A main result of this study is the composition of the metaphor "the eyes of the tree." This metaphor integrates research on scientific, philosophical and Native American forms of ecological knowledge and worldviews, and calls for conservation actions in a transdisciplinary way. This metaphor proposes a contextual view to observe, monitor, and understand the problematic of invasive species. It contributes to counteract the impact of exotic species through education and control practices that prioritize the ecosystem as a whole. In this way, it triggers approaches that are complementary to those that privilege of individual welfare over of the integrity of the biotic community
\end{abstract}

KEY WORDS: biocultural homogenization, Environmental Field Philosophy, ILTER, invasive species, LTSER.

\section{INTRODUCCIÓN}

Rápidos cambios socio-ambientales están ocurriendo a nivel global en el denominado Antropoceno, una nuevaerageológicacaracterizada por la prevalencia de transformaciones a escala planetaria causadas por el ser humano (Steffen et al. 2011). Entre los diversos cambios globales de esta nueva era se destaca la acelerada tasa de pérdida de biodiversidad, siendo las especies invasoras una de sus principales causas (Bellard et al. 2016; Vitousek et al.1997). Las especies invasoras son especies que han sido recientemente introducidas por el ser humano en áreas donde históricamente estaban ausentes y donde rápidamente han expandido su distribución generando impactos ecológicos y/o económicos (Catford et al. 2009).
Poblaciones humanas han estado transportando especies de un sitio a otro desde tiempos prehistóricos, tal como lo documentan las introducciones de cerdos, perros y ratas en islas del Océano Pacífico desde Oceanía probablemente desde antes del año 1000 AC (Steadman, 2006). Un aceleramiento en la tasa de introducciones intencionales y no intencionales de muchas especies tuvo lugar durante el proceso de la conquista europea de América iniciado en 1492 (Crosby Jr., 1972). Un aceleramiento más marcado en la tasa de movimiento global de especies tuvo lugar luego de la Revolución Industrial. Los avances tecnológicos en el transporte a nivel global, asociados con una economía global creciente y dominante, que demanda además el transporte de insumos entre múltiples destinos de la cadena de 
producción internacional, conllevaron a mayores oportunidades para la transferencia de especies de manera intencional o accidental desde sus zonas de origen hacia nuevos territorios (Essl et al. 2015).

El movimiento de algunas especies alrededor del mundo mediado por la actividad humana está generando un proceso denominado "homogeneización biótica" (McKinney \& Lockwood, 1999; Simberloff, 2013). Si bien la gran mayoría de las especies introducidas no logran establecerse en los nuevos ambientes (Williamson, 1996), muchas otras logran adaptarse a las nuevas condiciones $y$, entre ellas, algunas se vuelven especies invasoras. Estas especies invasoras pueden afectar la biología, abundancia y comportamiento de las especies nativas y la estructura y funcionamiento de todo un ecosistema (Ehrenfeld, 2010; Vilà et al. 2011). La degradación continua del ambiente resulta finalmente en diferentes ecosistemas globales dominados por un mismo grupo de animales $y$ plantas (Simberloff, 2013).

Adicionalmente, Ricardo Rozzi describe otro proceso similar de homogeneización que ocurre a nivel cultural. Los hábitos culturales y cosmovisiones locales de las personas, gestados en una profunda conexión con los hábitats y comunidad de cohabitantes locales, van siendo reemplazados por hábitos propios de la cultura capitalista occidental, con la consiguiente pérdida de culturas originales (amerindias o equivalentes), pérdida de lenguajes y concentración de la población humana en ciudades (Rozzi, 2012). Si bien estos dos procesos de homogeneización han sido estudiados de manera independiente por ecólogos y sociólogos, Rozzi (2012) propone que la pérdida de diversidad biológica y cultural a distintos niveles, local, regional y global, no ocurren de manera aislada, sino en un proceso conjunto de retroalimentación que él denomina "homogeneización biocultural". La investigación de estos procesos requiere por lo tanto de un enfoque biocultural que acople ambas dimensiones.

Con el objetivo de contrarrestar la homogeneización biocultural, la ética biocultural (Rozzi, 2012), en línea con "la ética de la tierra" de Aldo Leoplod (Leopold, 1949), la "ecología profunda" de Arne Naess (Naess, 1973) y la ética ambiental de muchas otras culturas, extiende la comunidad moral más allá del humano para incluir también otros seres vivos con los que cohabitamos el planeta. Sin embargo, la ética biocultural también hace una demanda dentro de la comunidad moral para incluir las poblaciones humanas marginadas que son oprimidas por formas de gobernanza principalmente asociadas al sistema de libre mercado neoliberal (Rozzi, 2012). Específicamente, defiende la conservación de los hábitats y el acceso a ellos por parte de las comunidades de co-habitantes (humanos y otrosque-humanos) como condición de posibilidad para la continuidad de sus hábitos de vida y bienestar (Rozzi, 2015a, 2015b). Estas "3 Hs" (hábitats, co-habitantes, hábitos) definen el marco formal de la ética biocultural, que es contextual y sistémica. Integra los dominios biofísico y cultural en conjunto con el socio-político, institucional y tecnológico. El dominio biofísico es común a los seres humanos y otros-que-humanos (esto es, aquellos componentes bióticos y abióticos de la biósfera). El simbólicolingüístico cultural es humano y es decisivo en los modos en que diversas culturas se valoran y relacionan con los seres otros-que-humanos. Estas relaciones eco-sociales se despliegan a través de instituciones que incluyen dimensiones cognitivas, normativas y tecnológicas.

Una de las formas institucionales más importantes para monitorear y eventualmente orientar el cambio socio-ambiental global necesario, es la red Internacional de Estudios Ecológicos a Largo Plazo (ILTER) ${ }^{1}$ (Li et al. 2015). Esta red incluye también redes de estudios socio-ecológicos a largo plazo (LTSER) ${ }^{2}$, tales como la red LTSER-Chile (Armesto et al. 2014). Estas redes constituyen una novedosa manera de monitorear las relaciones entre las dimensiones biofísicas con las dimensiones culturales a nivel global. Sin embargo, existe una falta de metodologías que permitan vincular los registros de patrones y procesos ecológicos con los valores éticos y sociales en los estudios a largo plazo y las acciones de conservación (Rozzi et al. 2010, 2014). Para observar, registrar, comprender y
1 ILTER, sigla en inglés de International Long-Term Ecological Research
LTSER, sigla en inglés de Long-Term Socio-Ecological Research 
contrarrestar la homogenización biocultural, los monitoreos y estudios ecológicos a largo plazo actualmente presentan tres graves limitaciones: a) se centran casi exclusivamente en el ámbito biofísico y habitualmente no incluyen dimensiones culturales - en particular, éticas (Rozzi et al. 2010); b) existe un sesgo hacia los monitoreos y estudios científicos teóricos, previniendo frecuentemente que los sitios ILTER se involucren en acciones concretas de conservación transdisciplinarias, que incluyan interacciones con otras instituciones, actores y disciplinas para contrarrestar las causas próximas y últimas de los procesos de invasiones biológicas y de homogenización biocultural (Rozzi et al. 2014); c) más del $90 \%$ de los sitios de estudios ecológicos a largo plazo se ubican en el hemisferio norte (Li et al. 2015; Rozzi et al. 2012). Estas limitaciones dejan invisibles y con falta de acción local fenómenos biofísicos, culturales, institucionales y socio-políticos que directa o indirectamente están vinculados a la problemática de especies invasoras y homogeneización biocultural en el hemisferio sur.

La ética biocultural provee un marco conceptual para resolver las limitaciones anteriores. Este marco estimula un cambio institucional en los programas de investigación y educación de las redes ILTER a través de su propuesta metodológica de la Filosofía Ambiental de Campo (FILAC), la cual ha sido desarrollada en el sitio más austral de la red ILTER y la red LTSERChile: el Parque Etnobotánico Omora (de ahora en adelante: Parque Omora; Rozzi et al. 2010). La FILAC ofrece un enfoque para integrar las dimensiones biofísicas, culturales e institucionales que no se limita al estudio de los fenómenos, sino que los integra en programas de acción transdisciplinaria de conservación y educación. Para integrar estas dimensiones, la aproximación metodológica de la FILAC utiliza un ciclo de cuatro pasos: 1) investigación interdisciplinaria ecológica y filosófica, 2) comunicación poética a través de la composición de metáforas y relatos, 3) actividades de campo con una orientación ética y ecológica, y 4) conservación in-situ. Desde el año 2008, la FILAC se ha desarrollado en el Parque Omora a través de diversos estudios académicos con trabajo interdisciplinario, proponiendo novedosas actividades con implicancias en educación, conservación y ecoturismo (Rozzi et al. 2008a, 2010, 2014). La FILAC ha comenzado a implementarse en otros sitios de la red ILTER como México y Brasil (Aguirre, 2014; Berches et al. 2015).

A partir del enfoque conceptual de la ética biocultural y la aproximación metodológica de la FILAC, el objetivo principal de este trabajo es contribuir a superar las tres limitaciones mencionadas anteriormente y que fueran identificadas para los actuales programas en los sitios de la red ILTER. En base al trabajo de la tesis doctoral de Ramiro D. Crego desarrollada en el Parque Omora sobre una especie invasora que ha arribado recientemente al extremo austral de América, el visón norteamericano (Neovison vison) proponemos: 1) integrar componentes ecológicos, filosóficos y tecnológicos en los estudios ecológicos a largo plazo, 2) ensayar una integración de la investigación científica con las cosmovisiones amerindias y acciones de conservación in-situ de modo transdisciplinario, 3) y contribuir a compensar el marcado sesgo geográfico hacia latitudes templadas y subárticas del hemisferio norte, a través de nuestro trabajo desarrollado en la ecorregión subantártica de Magallanes, que se ubica dentro del ámbito latitudinal menos incorporado en las redes ILTER del hemisferio sur. A partir del estudio de caso, proponemos una mirada más contextual y sistémica para percibir, registrar y comprender la problemática de las especies invasoras, la complejidad de sus efectos en los ecosistemas y culturas nativas, y contrarrestar su impacto mediante acciones de educación y control que priorizan el ecosistema como un todo, complementando enfoques valóricos que privilegian el bienestar de cada individuo por sobre la integridad de la comunidad biótica.

\section{UN ENFOQUE DESDE LA FILAC PARA ABORDAR A LAS ESPECIES INVASORAS EN UN SITIO LTSER SUBPOLAR}

El trabajo se desarrolló entre 2014 y 2015 en el Parque Omora $\left(55^{\circ} \mathrm{S}\right)$ y otros sitios de la isla Navarino y brazo noroeste del Canal Beagle. Estos sitios se ubican dentro de la Reserva de la Biósfera Cabo de Hornos (RBCH), la cual protege el extremo 
austral de la ecorregión subantártica de Magallanes (Fig. 1). El Parque Omora representa el centro científico de la $\mathrm{RBCH}$ y es sitio cofundador de la red LTSER-Chile, inaugurada en el parque en el 2008 (Rozzi et al. 2008b). Su enfoque de trabajo se ha centrado en la integración de las ciencias ecológicas y la ética ambiental en la educación y conservación biocultural, incluyendo el ecoturismo. La integración de la ciencia y la ética es especialmente relevante para enfrentar la problemática asociada a las especies invasoras, puesto que el manejo de las mismas frecuentemente involucra juicios de valor relacionados a la eliminación de individuos y poblaciones (Simberloff, 2012).

Basados en el enfoque de la conservación biocultural, proponemos una perspectiva innovadora con la cual abordar la problemática de las especies invasoras con un estudio de caso del visón norteamericano, una de las más recientes introducciones en el Parque Omora e isla Navarino. Mediante el uso de la FILAC como metodología de trabajo, analizamos primero la problemática desde los ámbitos biofísicos, filosóficos e institucionales. En base a esto, luego proponemos e implementamos una metáfora y actividades de campo que buscan conciliar las controversias de manejo en pos de la conservación de la diversidad biológica y cultural de la $\mathrm{RBCH}$.

Paso 1: Investigación ecológica y filosófica de la especie invasora, el visón norteamericano, y las problemáticas de conservación

Enelámbitobiofísico, la regiónsubantárticade Magallanes, ubicada en el sudoeste de Sudamérica, constituye el equivalente latitudinal más similar a

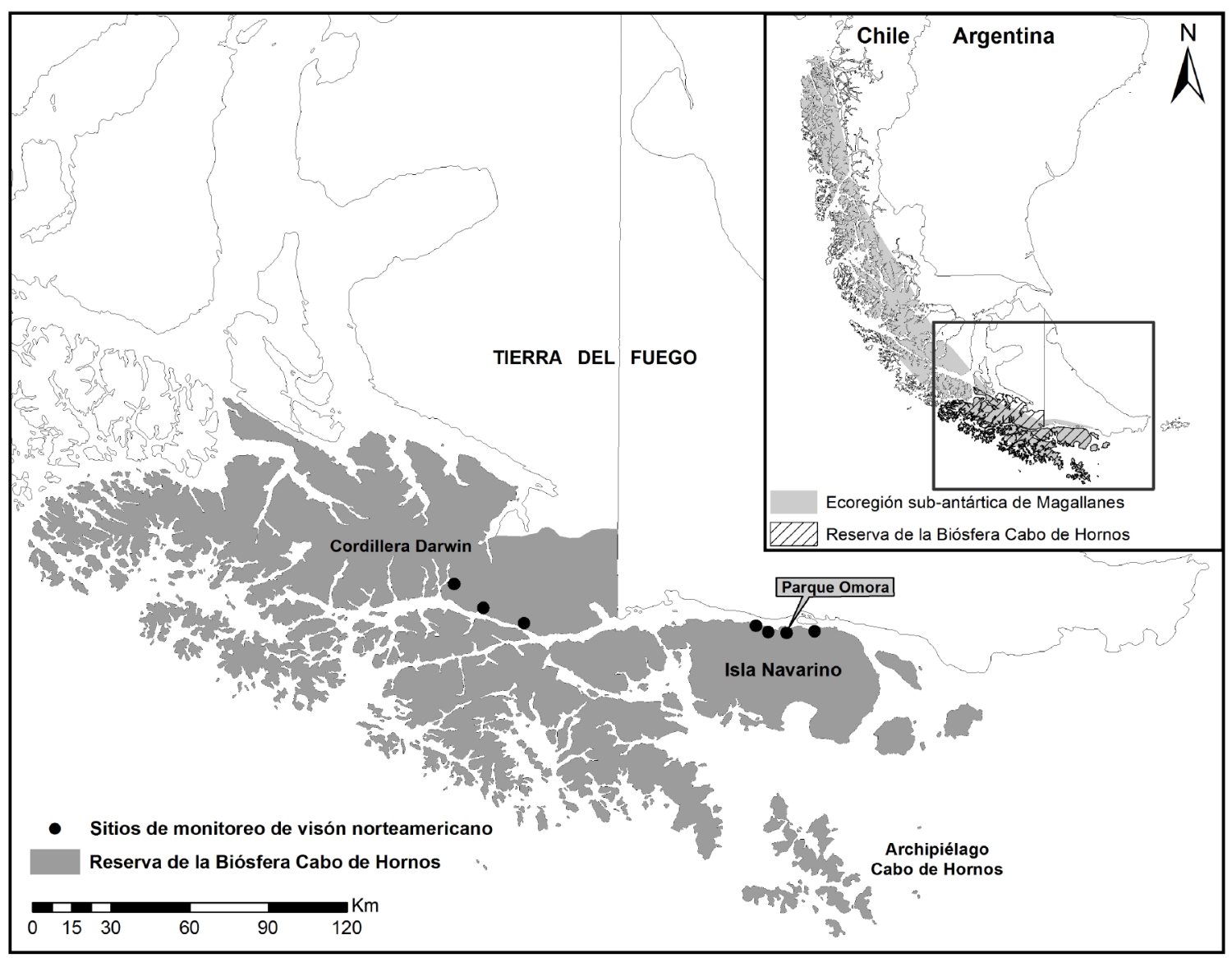

Fig. 1. El mapa muestra la Reserva de la Biosfera Cabo de Hornos, Chile, la localización geográfica del Parque Etnobotánico Omora (sitio LTSER más austral del mundo), con los sitios donde se monitoreó la presencia de visón norteamericano mediante el uso de cámaras trampa durante la tesis doctoral de Ramiro D. Crego, entre los años 2014 y 2015. 
la región subártica en el hemisferio norte. Si bien ambos hábitats subpolares presentan diferencias marcadas biofísicas y culturales, los nuevos hábitos culturales establecidos por los conquistadores europeos promovieron la reciente introducción de varias especies foráneas a la región. El mercado de pieles llevó a la introducción de especies peleteras, como son el castor norteamericano (Castor canadensis), la rata almizclera (Ondatra zibethicus) y el visón norteamericano (Jaksic et al. 2002). Esto determinó la introducción de un ensamble de "co-habitantes subárticos" provenientes de Norteamérica, que han reconfigurado la estructura y curso evolutivo de los co-habitantes, hábitos de vida y hábitats en el subantártico de Sudamérica (Crego et al. 2016).

El visón es un mustélido de hábitos semiacuáticos (Larivière, 1999) que fue inicialmente introducido desde granjas en Tierra del Fuego a partir de la década de 1940. La especie comenzó a ser abundante a lo largo de la costa del Canal Beagle en la década de 1990, detectándose por primera vez en la isla Navarino en el año 2001 (Rozzi \& Sherriffs, 2003), así como también en años posteriores en otras islas de la $\mathrm{RBCH}$ : isla Hoste (Anderson et al. 2006), isla Lennox (Davis et al. 2012), islas Gordon y Gabriel (Valenzuela et al. 2014) y al sur de los glaciares de la cordillera Darwin, Tierra del Fuego (Crego et al. 2015).

Al llegar a la isla Navarino, el visón comenzó a formar parte de una comunidad biótica que hasta ese momento había evolucionado libre de depredadores mamíferos terrestres. Los visones son animales de hábitos generalistas respecto al uso de hábitat y de la dieta, por lo tanto son capaces de adaptarse a la disponibilidad de presas (Larivière, 1999). En isla Navarino los visones consumen activamente aves y roedores (Schüttler et al. 2008; Ibarra et al. 2009; Crego et al. 2016). Como resultado de la plasticidad trófica y establecimiento del visón en el área, numerosas especies de aves se han visto negativamente afectadas por la nueva presión de depredación. Por ejemplo, el pato vapor no volador (Tachyeres pteneres) y el caiquén (Chloephaga picta) sufren una gran depredación de sus nidos por el visón (Schüttler et al. 2009; Liljesthröm et al. 2013). Además, los visones depredan sobre aves de bosque (Schüttler et al. 2008; Ibarra et al. 2009). Entre las aves de bosque, la dieta del visón incluye al carpintero magallánico (Campephilus magellanicus) (Jiménez et al. 2014; Crego et al. 2016), una especie considerada emblemática y de suma importancia por su papel ecosistémico y cultural a nivel regional (Arango et al. 2007).

El efecto directo del visón sobre las presas puede agravarse debido a que las especies de vertebrados nativos parecen ser ingenuas al riesgo de depredación. Podría esperarse que años de coexistencia hagan que las presas desarrollen comportamientos anti-depredatorios, pero si el tiempo no es suficiente, podrían ocurrir extinciones locales. Por ejemplo, las poblaciones de roedores nativos se han visto reducidas de manera significativa en los últimos 15 años (Crego et al. 2014). Estudios recientes sugieren que los ratones son incapaces de percibir al visón como un riesgo de depredación (Crego et al. 2018).

En el ámbito cultural (simbólico-lingüístico), observamos un proceso global de homogeneización biocultural que ha acarreado una problemática de conservación de la biodiversidad local de la $\mathrm{RBCH}$. La industria peletera fue un fenómeno económico y cultural global que introdujo en el extremo sur de Sudamérica especies de valor peletero durante el siglo XX. Estas especies invasoras, como el visón, han generado marcados efectos negativos sobre los ecosistemas subantárticos. Sin embargo, las medidas de control que pudieran aplicarse sobre tales especies invasoras generan controversias, las cuales han motivado acusaciones de xenofobia (e.g., extirpación, exterminio), uso de terminología bélica (e.g., enemigos naturales, armas novedosas), nativismo y racismo (Simberloff, 2012). Estas controversias en el ámbito de las especies invasoras inciden en la decisión de implementar o no medidas de control. La toma de decisiones ambientales referida a problemáticas tales como el control de especies invasoras, está culturalmente influenciada por la aceptación pública. A su vez, el modo en que una sociedad responde a la problemática, está relacionada a los "lentes conceptuales" con los cuales observa el problema. En este trabajo ensayamos una visión de la conservación de la biodiversidad de la $\mathrm{RBCH}$ que forja nuevos "lentes conceptuales" basados en las ciencias ecológicas y evolutivas, cosmovisiones amerindias y ética biocultural. 
De acuerdo a la ciencia, la diversidad global está en gran medida dada por la diversidad y dinámica de hábitats donde las especies evolucionan. El aislamiento geográfico determina que, en el curso de largos lapsos de tiempo, las especies diverjan hasta formar otras nuevas. De este modo, en los bosques templados del sur de Sudamérica, aislados de otros bosques por al menos 1500 a $2000 \mathrm{~km}$ de ambientes séricos y océanos, evolucionaron numerosas especies que hoy son endémicas a la región (Armesto et al. 1998). Bajo esta narrativa científica, la biodiversidad singular de estos bosques se ha visto "invadida" por nuevos co-habitantes introducidos a partir de los procesos de reciente colonización europea, que a su vez han gatillado un proceso de homogeneización biocultural. Estos procesos no solo afectan la diversidad actual, sino que también comprometen los procesos evolutivos y sus derivas futuras (Mooney \& Cleland, 2001; Sarrazin \& Lecomte, 2016).

Las cosmovisiones amerindias en las regiones subpolares también son únicas, producto de miles de años de co-habitar determinados hábitats y en íntima relación con determinados co-habitantes. De acuerdo a la cosmogonía Yagán, pueblo originario que ha habitado en la región de la $\mathrm{RBCH}$ desde hace milenios, los mamíferos, las aves y otros animales descendían de seres humanos. Esta visión evolutiva precolombina implica una relación de parentesco entre los seres humanos y el conjunto de co-habitantes de los ecosistemas subantárticos (Rozzi, 2004). Una cosmogonía similar es albergada por los koyukon, quienes han habitado en el polo geográfico opuesto a los bosques subantárticos: en las márgenes de los ríos Koyukuk y Yukon de Alaska, en el subártico. De acuerdo a la cosmogonía de los koyukon, existe un Tiempo Lejano en el cual los animales y plantas eran humanos. En algún punto de ese Tiempo Lejano, ciertos humanos murieron $y$ se transformaron en animales o plantas. Por lo tanto, para los koyukon los animales y plantas son literalmente sus parientes, sus ancestros, hermanos, hermanas (Nelson, 1984). Esta cosmogonía ha orientado ecológica y éticamente la forma de habitar de los koyukon en sincronía con la estacionalidad, los procesos ecológicos y la comunidad de cohabitantes de los ecosistemas forestales subárticos, practicando la moderación y el respeto al medio ambiente, coexistiendo por miles de años (Nelson, 1984). Este sentido de parentesco y respeto por el hábitat es similar al implicado por la cosmogonía yagán (Rozzi et al. 2010).

Como señalamos anteriormente, un principio formal básico de la ética biocultural es que los hábitos de vida de las comunidades de co-habitantes están interrelacionados con los hábitats donde estos tienen lugar. Estas " $3 \mathrm{Hs"} \mathrm{de}$ la ética biocultural ofrecen un marco conceptual que permite una mejor identificación de grados de responsabilidad diferencial entre co-habitantes humanos en la génesis del Antropoceno-incluyendo la rápida homogenización biocultural-y una mayor valoración de la plétora de formas de co-habitar responsable y sustentable con la comunidad biótica en los ecosistemas (Rozzi, 2015a). La conservación de los hábitats y el acceso a ellos por parte de las comunidades de co-habitantes (humanos y otrosque-humanos) es la condición de posibilidad de la continuidad de su vida; se convierte en un imperativo ético que debe ser incorporado en las políticas de desarrollo como una cuestión de justicia eco-social (Rozzi, 2015b).

Proponemos entonces que por conservación se entiende preservar el curso evolutivo del hábitat, los co-habitantes y sus hábitos. Estos cursos evolutivos han conducido a estos sitios a ser únicos, albergando una diversidad biótica y cultural distintiva y diferente de otras regiones del mundo. En otras palabras, esta conservación evita la homogenización biocultural, respetando la diversidad de las biotas nativas y sus hábitos de vida. Esto implica una visión holística, incorporada por la ética ambiental eco-céntrica que valora la integridad del ecosistema como eje principal de conservación (Palmer, 2013). En el ecosistema se protegen las intrincadas relaciones entre los cohabitantes nativos que dieron forma a la biodiversidad, y también a las cosmovisiones de los pueblos originarios antes de la introducción de las especies no-nativas, valorando al ecosistema como un todo y no solo a los individuos.

Finalmente es necesario enmarcar la discusión dentro del ámbito institucional. El Antropoceno tiene sus causas últimas en valores éticos, formas culturales y transformaciones institucionales que han conducido a que la economía moderna se haya desvinculado de la consideración de contextos 
biofísicos y, más recientemente, de contextos sociales. Sin embargo, el modelo económico reinante gravita hoy sobre la gobernanza a nivel local, regional y mundial. El Parque Omora procura revincular la economía, la ciencia, la cultura, la sociedad y lo biofísico mediante un programa de investigación, educación y conservación biocultural. En nuestro estudio sobre las especies invasoras, trabajamos en conjunto con el Servicio Agrícola y Ganadero (SAG) y la Corporación Nacional Forestal (CONAF). De esta manera, no solo integramos distintas disciplinas, sino también distintas instituciones, estableciendo un trabajo transdisciplinario y no solo interdisciplinario. Esta colaboración interinstitucional es fundamental para proyectar programas de control de especies exóticas y de conservación en el largo plazo (Rozzi et al. 2006). El Parque Omora lidera también, la integración de la ética y las ciencias en los programas de educación de la red ILTER. Este marco institucional facilita que las metodologías y resultados de nuestros estudios particulares en isla Navarino puedan ser aplicados y adaptados en otros sitios de la red ILTER que están sujetos a presiones análogas de las especies de mamíferos exóticos invasores en otras regiones del mundo.

Paso 2. Composición de metáforas y comunicación mediante narrativas: "Los ojos del árbol"

La composición de metáforas es una parte esencial de la FILAC para comunicar conceptos al público general y también, para integrar el estudio ecológico y filosófico a través de analogías que sintetizan valores y conceptos en conservación y ética biocultural (Rozzi et al. 2010). En este trabajo, compusimos la metáfora con el objetivo de combinar los conocimientos biofísicos, simbólicolingüísticos y tecnológicos. La metáfora propone un entendimiento y valoración más holístico del funcionamiento de los ecosistemas y las disrupciones causadas en estos por la introducción de especies invasoras y, en particular, la del visón en isla Navarino.

Las ciencias ecológicas y evolutivas caracterizan a la isla Navarino como un área libre de mamíferos depredadores terrestres, por lo tanto la fauna y sus interacciones ecológicas han evolucionado libres de la presión de depredación que representa el visón. A diferencia de las aves del bosque, que fácilmente pueden ser avistadas, los visones son animales de hábitos nocturnos, elusivos y difíciles de observar. Sin embargo, una innovación tecnológica, la cámara trampa, provee de una herramienta novedosa y eficaz con la cual los investigadores pueden registrar, estudiar y comunicar la actividad de este tipo de especies (O'Connell et al. 2011). Estas cámaras se suelen instalar en la base del tronco de un árbol. Los árboles del género Nothofagus que hoy conforman los bosques subantárticos de Magallanes son longevos $y$ han estado presentes mucho antes que los visones arribaran a la isla. Dentro del Parque Omora existen coihues de Magallanes (Nothofagus betuloides) de entre 202 y 350 años de edad (Lombardi et al. 2011; Llancabure, 2011), mientras que nuevos estudios documentan edades de hasta 400 años (A. Gutiérrez com. pers.). Estos individuos son "testigos vivos" de la historia de la zona, del proceso que siguió al arribo de los europeos al Cabo de Hornos, el establecimiento de las prácticas y creencias occidentales, entre ellas, las especies domésticas y la industria peletera. Como resultado de esta historia reciente, en isla Navarino hoy habitan vacas, caballos, cerdos, perros y gatos baguales, y también un ensamble de mamíferos originario de Norteamérica, el castor, la rata almizclera y el visón, que hoy interactúan y afectan negativamente la estructura de los ecosistemas (Crego et al. 2016).

De acuerdo a la cosmovisión de los koyukon, los arboles del bosques están siempre observando y juzgando la conducta de los cazadores y cada miembro de la comunidad (Nelson, 1984). Los koyukon nunca caminan solos en el bosque: es "como si los árboles tuvieran ojos". Las cámaras trampa utilizadas para estudiar el visón en isla Navarino, materializaron con un enfoque científico, pero de algún modo análogo, la cosmovisión koyukon, adosando "ojos tecnológicos" a los árboles. Estas trampas permitieron detectar una expansión en los hábitos de vida del visón, desde los característicos hábitos subacuáticos en el territorio de los koyukon, hacia hábitos más terrestres en Navarino (Fig. 2; Crego et al. 2018b). La ética biocultural afirma que los hábitos de vida se interrelacionan con las comunidades de co- 


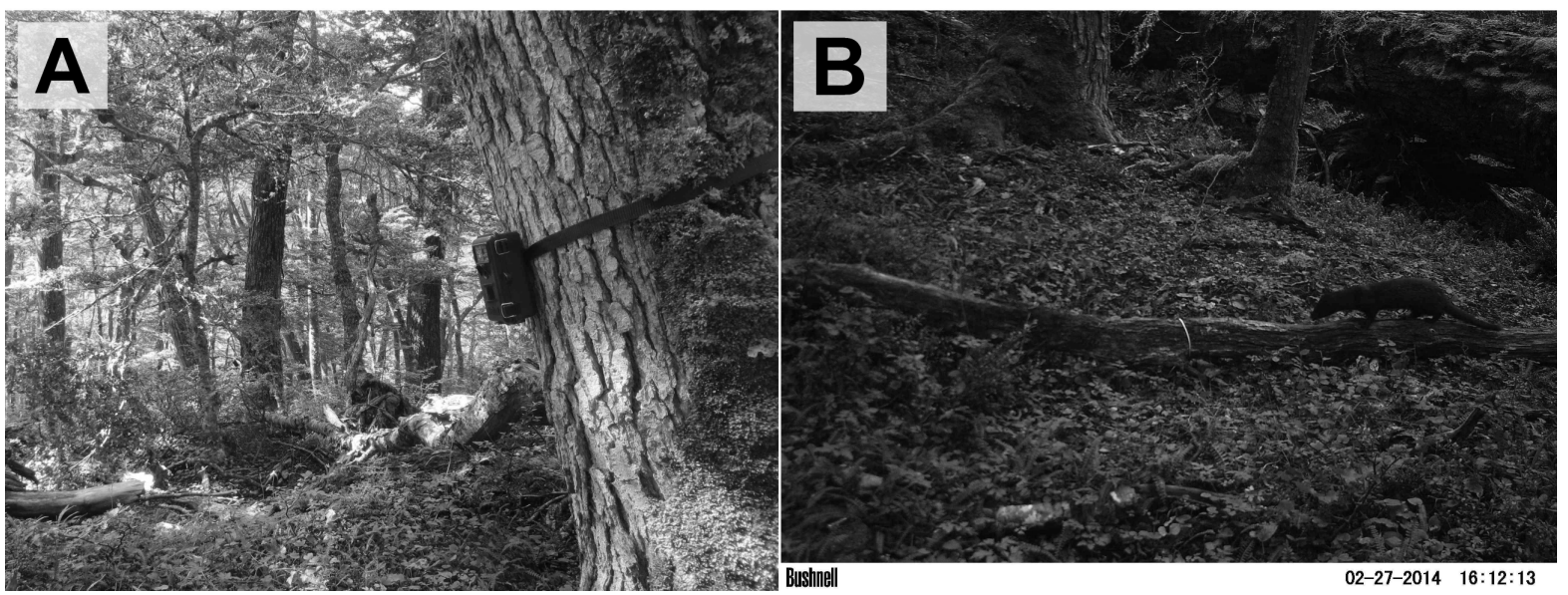

Fig. 2. Cámara trampa instalada en la base de un coihue Magallánico en isla Navarino, Chile (A) y fotografía de un individuo de visón norteamericano tomada por la misma (B).

habitantes y los hábitats donde estos se despliegan. En nuestro caso, el "co-habitante árbol" ha sido dotado de un "tecno-ojo" que permite registrar el arribo de un nuevo co-habitante, que gesta el dilema ético de cómo co-habitar y juzgar la conducta humana en esta nueva relación de cohabitación. Desde estas tres fuentes, científica, amerindia y ética, compusimos la metáfora "los ojos del árbol".

\section{Paso 3. Actividades de} campo ecológica y éticamente guiadas: Conectando los ojos al árbol.

El programa de educación del Parque Omora enfatiza la importancia de los encuentros directos con la naturaleza para lograr estimular la percepción y valoración de la diversidad biológica y cultural (Rozzi et al. 2010). Para comprender y comunicar experiencialmente la metáfora los ojos del árbol se diseñaron dos tipos de actividades de campo guiadas de manera ética y ecológica: a) una visita a una nueva estación llamada "Los ojos del árbol" dentro del sendero interpretativo "Los bosques más australes del planeta" en el Parque Omora, y b) un taller y concurso de fotografía con la comunidad de Puerto Williams.

Los senderos interpretativos que se visitan en el Parque Omora tienen estaciones de observación. Basados en nuestra investigación, creamos una nueva estación que invita a conocer los "lentes tecnológicos" (la cámara trampa) y cómo estos lentes se integran a la ILTER y la LTSER a través de la FILAC (Fig. 3). A partir de una visita ecológica y éticamente guiada, los visitantes indagan sobre los "lentes tecnológicos" conociendo el instrumento (cámara trampa) y sus registros. Los registros fotográficos revelan la existencia del visón, cuya presencia permanece de otro modo invisible a los visitantes. También dejan de ser invisibles las interacciones bióticas y el impacto del visón sobre la biota nativa y el ecosistema.

La visita guiada a través de los ecosistemas de bosques subantárticos de Magallanes, incluye también un "lente conceptual" que permite visualizar más nítidamente a los co-habitantes del bosque, sus hábitos de vida y la fragilidad de los ecosistemas forestales más australes del planeta frente al arribo de las especies invasoras. Una indagación sobre los "lentes bioculturales" permite además conocer las narrativas koyukon que inspiran la metáfora. Esto se complementa con las narrativas yaganes que tienen lugar durante las visitas al Parque Omora. De esta manera, los "lentes tecnológicos" junto a los "lentes conceptuales y bioculturales" permiten a los visitantes, autoridades, profesores y otros miembros de la comunidad, generar una imagen mental para poder comprender y valorar los complejos procesos socio-ecológicos y éticos que implican la llegada del visón al ecosistema subantártico.

La práctica de la FILAC en el Parque Omora requiere también la participación de diversas instituciones y miembros de la comunidad. Para 
implementar una aproximación transdisciplinaria, desarrollamos un concurso y taller fotográfico. El objetivo fue extender el "lente tecnológico" de los ojos del árbol a las cámaras fotográficas utilizadas por los miembros de la comunidad. De este modo se buscó incentivar a la población local a tener experiencias de contacto "cara-a -cara" con la comunidad de co-habitantes de los ecosistemas subantárticos. Además, la actividad involucró a las instituciones gubernamentales y empresas locales (que proveyeron los premios), en la problemática de las especies invasoras. En una primera etapa, realizamos una serie de talleres abiertos a la comunidad, integrando contenidos sobre invasiones biológicas en isla Navarino y fotografía. En una segunda etapa, las personas participaron en un concurso fotográfico con la temática de especies invasoras de la isla. En una tercera etapa, hemos utilizado las redes sociales para continuar, incluso hasta hoy en día, difundiendo conocimiento sobre la temática ${ }^{3}$. La importancia de la actividad no radica en el premio material, sino en promover actividades de experiencia directa con la naturaleza, que utiliza tanto "lentes tecnológicos" como "lentes conceptuales" como herramienta para ampliar el "lente biocultural" con el cual la naturaleza es percibida, valorada y cuidada. Para la red ILTER, esta actividad expande las metodologías de investigación y conservación hacia formas de ciencia ciudadana.

\section{Paso 4. Conservación in situ: Estación interpretativa "Los ojos del árbol"}

En un sentido poético, la estación de los ojos del árbol ilustra un programa de monitoreo de largo plazo para la conservación de la biodiversidad biológica y cultural nativa. Se eligió uno de los árboles de mayor tamaño dentro del sendero "Los bosques más australes del planeta" para instalar una cámara trampa demostrativa en el Parque Omora. El árbol de lenga (Nothofagus pumilio) elegido, evoca a través del gran diámetro de su tronco una prolongada longevidad, que le ha permitido ser testigo de las interacciones entre los co-habitantes del bosque desde centenas de años, que preceden a la llegada del visón a la $\mathrm{RBCH}$ (Fig. 3). Esta estación evoca en el visitante la imaginación de largas escalas temporales propias de los ecosistemas cuya existencia excede a las breves vidas humanas. Frente a esta centenaria lenga, el visitante es invitado a transitar desde una visión individual a una visión ecosistémica. La cámara adosada al tronco del árbol, permite a los visitantes comprender una metodología científica para conducir monitoreos y estudios ecológicos a largo plazo. El visitante también comprende la relevancia de la red LTSER en cuanto genera instituciones y programas de investigación y conservación cuya extensión excede a la vida de cada proyecto y de cada investigador individual (Rozzi et al. 2014). En los científicos, la metáfora estimula la comprensión y cultivo de un sentido de responsabilidad que trasciende el estudio de corto plazo y los estudios teóricos. Se supera la relación de utilización de la naturaleza como "objeto de estudio" y se fomenta el sentido de responsabilidad ética con la comunidad de co-habitantes, inspirada por la ciencia y guiada por la ética. Desde 2015, esta estación se ha incorporado al sistema de senderos interpretativos que el Parque Omora tiene implementado para la realización de actividades educativas y ecoturísticas y que han sido diseñados usando la aproximación metodológica de la FILAC (Rozzi et al. 2008a, 2010, 2014). Esta comunidad no ha sido solo estudiada, sino que también se ha asumido una acción que transforma su realidad, procurando el bienestar de los co-habitantes y la conservación de sus hábitos y hábitats.

\section{LOS OJOS DEL ÁRBOL: UNA VISIÓN ECOLÓGICA Y DE ÉTICA ECOCÉNTRICA}

Como señalamos anteriormente, a pesar de los marcados efectos negativos que muchas especies invasoras generan en los ecosistemas invadidos, el control de las mismas genera serias controversias sociales. Diversas campañas para controlar y mitigar los efectos negativos de las especies invasoras se han desarrollado en Chile y distintas partes del mundo. Sin embargo, tales campañas han sido históricamente repudiadas por movimientos de derecho de los animales (Simberloff, 2012). Es importante resaltar que tales movimientos surgen en defensa de animales vertebrados, y en un par de casos, de especies de árboles (ver Simberloff, 


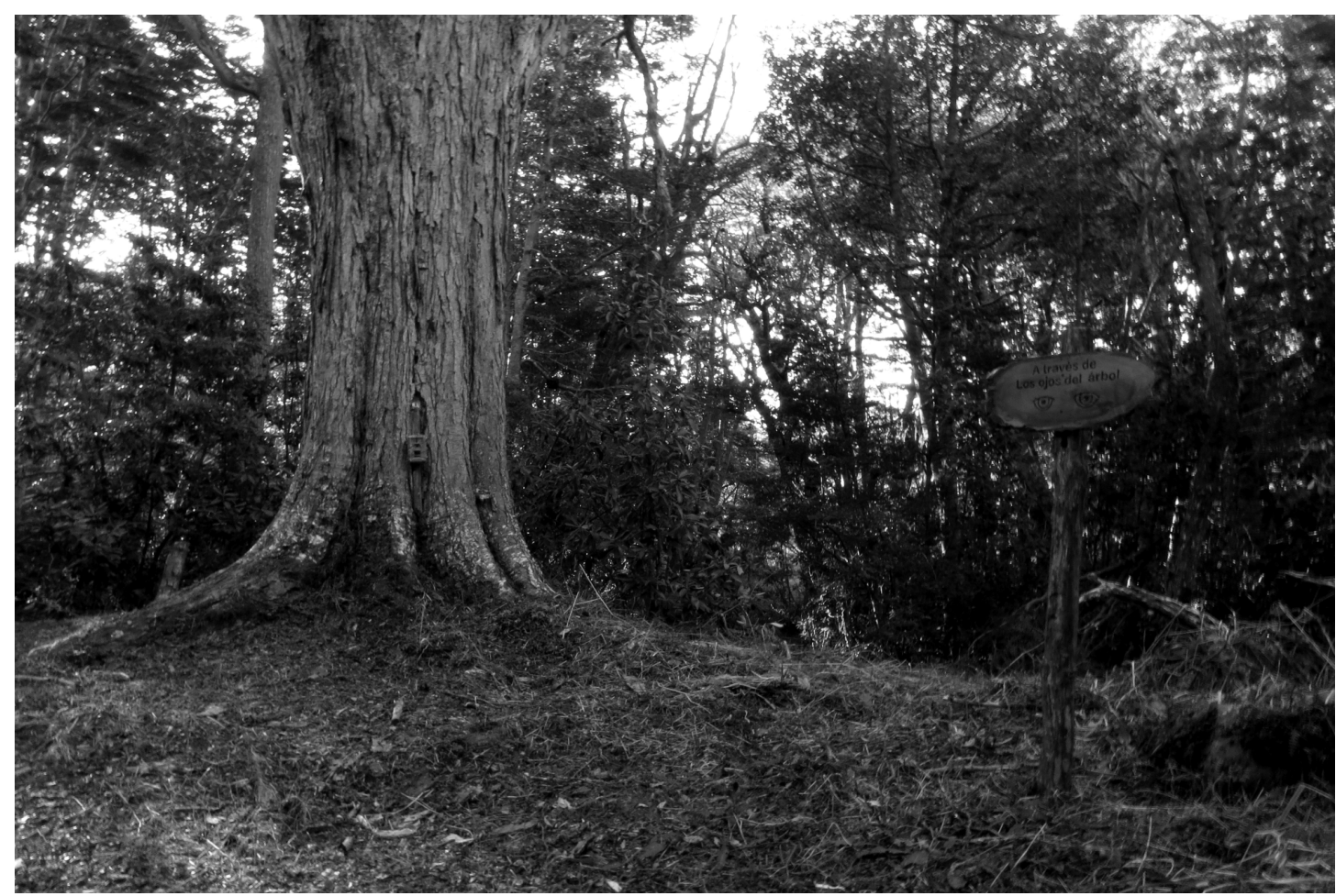

Fig. 3. Estación de los ojos del árbol ubicada en el Parque Etnobotánico Omora. La estación ilustra "el ojo tecnológico" utilizado en un programa de monitoreo de largo plazo para la conservación de la diversidad biológica y cultural nativa en el sitio ILTER y LTSER más austral del mundo. La cámara trampa demostrativa se encuentra adosada a la base de un árbol de lenga centenario que ha sido testigo de las interacciones entre los co-habitantes del bosque que preceden a la llegada del visón norteamericano a la Reserva de la Biósfera Cabo de Hornos.

2012). La empatía con las especies suele disminuir hacia especies no carismáticas y taxonómicamente menos emparentadas con los humanos, tales como insectos y plantas herbáceas o no vasculares. Es relevante entonces examinar la discusión sobre la consideración moral del control de organismos otros-que-humanos.

La extensión de la consideración moral hacia seres otros-que- humanos se ha sistematizado en una reciente subdisciplina filosófica, la ética ambiental, que analiza, orienta y evalúa el accionar humano sobre el medio ambiente (Hargrove, 1989). Esta subdisciplina filosófica se introdujo en la academia anglosajona en los años 1960 y 1970 en Estados Unidos, cuestionando principalmente el sesgo antropocentrista en la ética de la cultura occidental, considerando necesaria la extensión moral hacia seres otros-que-humanos y la naturaleza como un todo. Gran influencia sobre esta subdisciplina ha tenido el pensamiento de Aldo Leopold y su ensayo "la ética de la tierra", donde argumenta que la raíz de los problemas ambientales es de naturaleza filosófica y proclama un cambio en el papel del Homo sapiens, desde conquistador de la tierra hacia simple ciudadano de ella (Leopold, 1949). Más radicales son las ideas de la "ecología profunda" propuestas en un principio por el filósofo noruego Arne Naess, quien expresó la necesidad de equiparar los valores morales humanos con los del medio ambiente, proponiendo profundos cambios políticos, sociales y económicos para lograr el cohabitar humano con el resto de los seres vivos (Naess, 1973). Estos movimientos son catalogados como holismo ecocéntrico o ecocentrismo (Palmer, 2013). Contemporáneo a la gestación de la ética ambiental se afirmó el movimiento de la liberación animal y de los derechos de los animales, en gran medida liderados por los filósofos Peter Singer en Australia (Singer, 1975, 1993) y Tom Regan en los Estados Unidos (Regan, 1975). Basados en una tradición de ética utilitarista que considera como correcto evitar el dolor y favorecer el placer en 
animales con capacidad de sentir, estos filósofos proclamaron una extensión de la consideración moral hacia organismos sintientes más allá de los seres humanos. A pesar de la similitud en la extensión de la comunidad moral, los movimientos de ética ambiental con el de liberación animal y derechos de los animales presentan algunas diferencias relevantes. Callicott (1980) ha argumentado que la ética ambiental es más inclusiva y holística que el movimiento de liberación animal y derecho de los animales: mientras que los dos últimos enfatizan una ética por los individuos, la ética ambiental se preocupa del valor y la integridad de la comunidad biótica en su conjunto.

Esta distinción individualista versus holista es de gran relevancia para el manejo de especies invasoras y la conservación de los ecosistemas. Las bases filosóficas en el sufrimiento de los animales sintientes, propias de los movimientos de liberación animal y derechos de los animales, entran naturalmente en conflicto con las prácticas de conservación (Hutchins \& Wemmer, 1986). Cualquier práctica de manejo de especies que implique la remoción de individuos para evitar efectos negativos a nivel comunitario o ecosistémico, viola el derecho a la vida de los animales. Si bien estas ideas son un aporte importante hacia la extensión moral más allá de los humanos, al enfocarse en la capacidad de sentir como los humanos, dejan de lado el resto de los seres vivos y los ecosistemas en general. Llamativamente, pareciera existir una correlación entre la vida en las urbes y la defensa de los derechos animales. Si bien ningún estudio confirma tal aseveración, este parece ser el caso en Chile. El organismo de gobierno encargado del control de especies invasoras en Chile es el Servicio Agrícola y Ganadero (SAG-FONDEMA, 2008). El SAG ha llegado a sufrir atentados en sus oficinas de Punta Arenas por parte de defensores de los animales (mamíferos sintientes) ${ }^{4}$ que se oponen a las medidas de control y erradicación de especies introducidas, como el visón y el castor y, en especial, los caballos y los perros baguales. Tales movimientos de defensa de los derechos de los animales, ocurren en áreas urbanas como Punta Arenas y Santiago, la capital de Chile, como en otras muchas ciudades del mundo. Sin embargo, en Puerto Williams, un pequeño poblado ubicado en isla Navarino donde la gente vive en constante contacto con la naturaleza, gran parte de la población apoya los planes de control, mostrando además preocupación por la disminución de las aves o desaparición de guanacos en la zona, debido a los visones invasores o los perros baguales (Schüttler et al. 2011).

El co-habitar con la diversidad y las experiencias cara-a-cara con la naturaleza son fundamentales para generar valores para la conexión con la naturaleza (Berghöfer et al. 2008). Esto es especialmente relevante para las personas que viven en grandes urbes, más alejadas de tales vivencias, en lo que hoy se conoce como "la extinción de la experiencia" (Soga \& Gaston, 2016). Hoy día, más del $50 \%$ de la humanidad vive en grandes urbes, por lo que la mayoría de las personas, y en especial los niños (Soga \& Gaston, 2016), adquieren su conocimiento de la diversidad biológica y cultural en contextos urbanos, física y emocionalmente lejos del contacto con los habitantes humanos y otros-que-humanos nativos de los ecosistemas (Rozzi, 2012). Las urbes son además, los centros de toma de decisiones sobre la conservación y donde la población influye más sobre los medios de comunicación, y estos, a su vez, sobre la toma de decisiones.

La metáfora de los ojos del árbol busca que las personas puedan, a través de un "ojo tecnológico" como el de la cámara, "ver" y "sentir" como "vería" y "sentiría" un árbol que ha vivido por centenares de años y ha sido testigo de la llegada e impacto del visón en el bosque subantártico de Magallanes, expandiendo los lentes "conceptuales y bioculturales". La empatía con el árbol, un ser no sintiente, genera una ética hacia la integridad del ecosistema, más allá de los individuos y hacia la cultura que supo desarrollarse en el hábito de co-habitar con los habitantes nativos. Una ética de respeto a los co-habitantes como la tienen los yoyukon y yagan. Esta ética con un entendimiento holista en la visión del ecosistema, busca generar una nueva línea de discusión para resolver el conflicto generado por los planes de control arraigado en los derechos de los animales. De este modo buscar reconciliar las diferencias en pos de la 
conservación de individuos nativos y el ecosistema en general, considerando no sólo a los mamíferos carismáticos, sino también a las pequeñas aves, los ratones, las plantas, los musgos, y todas las interacciones que ocurren en la comunidad de co-habitantes, complementando otras metáforas enmarcadas en la FILAC (Rozzi et al. 2014). Una ética que revaloriza la diversidad biológica y cultural y pone un límite a la homogeneización biocultural que resulta de adoptar desinformadamente como propias especies introducidas por una cultura hegemónica, que se incorporan rápidamente en las personas que habitan lugares incluso tan remotos como la isla Navarino.

\section{HACIA LA ECOLOGIA DE LAS EMOCIONES.}

Nuestra metáfora no se limita a "ver" como el árbol, sino que implica también "sentir". Kay Milton hace una excelente descripción de la necesidad de incorporar las emociones dentro la ecología para poder afrontar los problemas de conservación (Milton, 2002). Los que buscan conservar la naturaleza, proclamando la extensión de la consideración moral más allá de los humanos, lo hacen porque tienen una gran conexión emocional con los seres vivos y la naturaleza. Sin embargo, la tradición científica en gran medida basada en los trabajos de Descartes, ha puesto a las emociones en dicotomía con la racionalidad (Damasio, 1994). Sin duda, la relación entre emoción y racionalidad son de gran importancia en el debate de la conservación, que también incluye a los ecólogos. Milton argumenta que son las emociones las que operan en la conexión entre las personas y el medio ambiente. Las emociones permiten el desarrollo de la percepción y el conocimiento, y las emociones se gatillan con la experiencia directa. El deseo de conservar se hace explícito en la identificación con la naturaleza, los hábitats, los paisajes, los organismos otros-quehumanos y sus hábitos (Milton, 2002). Es en la experiencia con la naturaleza cuando es posible entender el conocimiento, para poder optar por una posición frente a los problemas ambientales y las metáforas combinadas con la experiencia pueden jugar un papel primordial en ese proceso.

Los "ojos del árbol" permiten entonces, mediante la experiencia directa, percibir al bosque en un contacto directo $y$, mediante la experiencia indirecta a través de un lente tecnológico, percibir lo que está oculto a nuestros sentidos. De ese modo, se disparan emociones que permiten un mejor entendimiento del problema real que las especies invasoras generan sobre la biodiversidad local, en el proceso de homogeneización biocultural. Es mediante la empatía con el árbol, que observa la evolución a través del tiempo de los co-habitantes del bosque, tanto animales como culturas nativas, que podemos entonces entender la importancia de conservar la riqueza biológica, evolutiva y cultural que pertenecen a un sitio único. Es mediante la metáfora y la experiencia que se abren los sentidos, que podemos entender el rol que la sociedad y el discurso hegemónico juegan en el sistema actual, y la importancia de transcender la ecología superficial hacia la ecología profunda. Tanto el ver y el comprender, como el sentir y el experienciar, guían las decisiones de conservación biocultural de la FILAC.

\section{RESOLVIENDO LAS LIMITACIONES EN LOS PROGRAMAS ILTER PARA OBSERVAR, REGISTRAR, COMPRENDER Y CONTRARRESTAR LA HOMOGENIZACIÓN BIOCULTURAL}

En este trabajo utilizamos la ética biocultural y FILAC como metodología de trabajo para proponer una metáfora que integra componentes tecnológicos, ecológicos, filosóficos y amerindios. Los "ojos del árbol" contribuyen a superar las limitaciones identificadas en nuestra introducción para los sitios ILTER: estudios focalizados en el ámbito biofísico ignorando los aspectos culturales, estudios meramente teóricos y con un marcado sesgo hacia el hemisferio norte. Nuestro trabajo no se basa solo en el estudio del ámbito biofísico, sino que también incluye las interrelaciones culturales y éticas entre el hábitat, la comunidad de cohabitantes y sus hábitos de vida. El trabajo parte del estudio de la problemática ecológica que implica la introducción del visón en los frágiles ecosistemas de los bosques subantárticos de Magallanes. La problemática es común a otros sitios del mundo, como lo es la dificultad de implementar prácticas de manejo y control que encuentran oposición en la visión de los derechos de los animales. 
Para lograr resolver este problema, se plantea la conservación de la biodiversidad basada en "lentes conceptuales" de las ciencias ecológicas y evolutivas, de cosmovisiones amerindias y de la ética biocultural.

En la metáfora de los ojos del árbol, el "lente tecnológico" permite expandir los lentes "conceptuales y bioculturales" al generar una empatía con un árbol que ha vivido por muchos años y es testigo vivo de los procesos de homogenización biocultural e impacto del visón en los co-habitantes del bosque. De este modo se integra la tecnología utilizada, las cámaras trampa, con una cosmovisión amerindia que concibe la capacidad de los árboles de "observar", propia de la cultura koyukon. La empatía con el árbol, genera una ética con un entendimiento holista en la visión del ecosistema para resolver el conflicto generado por los planes de control arraigado en visiones individualistas, para promover la conservación de todos los co-habitantes que conforman el ecosistema nativo. La metáfora motiva y orienta, a su vez, acciones transdisciplinarias, que incluyen interacciones con diversas instituciones, actores y disciplinas. La dimensión local se extiende desde el trabajo con la comunidad, hacia un mayor rango de público en el trabajo in-situ con una amplia diversidad de visitantes al Parque Omora. Además, a través de la red ILTER y LTSER se busca abarcar una escala global que integra las ciencias y la ética para contribuir a una continuidad de procesos ecológicos y evolutivos que sustenta la riqueza de la vida en el planeta. Así mismo, el trabajo llevado a cabo en los bosques más australes del mundocontribuye a contrarrestar el marcado sesgo hacia el hemisferio norte.

En conclusión, la metáfora de los ojos del árbol procura integrar el trabajo de investigación científica con las culturas de los pueblos originarios y de la sociedad contemporánea. Para poder afrontar los problemas ambientales actuales, se necesita que los ecólogos asuman un papel más activo en la sociedad integrando trabajos a escalas locales y globales. Arne Naess nos ha instado a superar la ecología superficial puramente tecnocientífica y forjar una ecología profunda que integre la cultura y las instituciones, con mayor acción y participación en la sociedad. Hoy, la FILAC desde el sur del mundo y la metáfora de "los ojos del árbol" proveen de un lente conceptual, ético y tecnológico para percibir, registrar y comprender la biota subantártica de Magallanes, para luego poder contrarrestar la homogenización biocultural.

\section{AGRADECIMIENTOS}

Queremos agradecer a las numerosas personas que colaboran con el programa de Filosofía Ambiental de Campo del Parque Etnobotánico Omora y al apoyo de los proyectos ICM P05-002 y Basal-CONICYT PFB-23 y Apoyo a Centros Científicos y Tecnológicos de Excelencia con Financiamiento Basal CONICYT AFB170008 del Instituto de Ecología y Biodiversidad de Chile (IEB) y FONDECYT 11130451 de la Universidad de Magallanes (UMAG), el Toulouse Graduate School de la University of North Texas y la Rufford Foundation. Queremos dar un agradecimiento especial al fotógrafo Gabriel N. Gómez quien colaboró de manera desinteresada con los talleres y cursos de fotografía y a la Ilustre Municipalidad de Cabo de Hornos y a la empresa EDELMAG por su colaboración con los premios del concurso. También agradecemos a Javiera Malebrán Muñoz, Carolina Baldini y Carolina Pérez Troncoso por sus comentarios y contribuciones al manuscrito. Este trabajo es una contribución del Programa de Conservación Biocultural Subantártica, conjuntamente coordinado por la UMAG, la Fundación Omora e IEB en Chile y la University of North Texas en EE.UU.

\section{BIBLIOGRAFÍA}

Aguirre, J. (2014). Hermeneutic and Field Environmental Philosophy to Integrate Ecological Sciences and Ethics into Earth Stewardship. In R. Rozzi, F. S. Chapin, J. B. Callicott, S. T. A. Pickett, M. E. Power, J. J. Armesto \& May Jr. (Eds.), Earth Stewardship: Linking ecology and ethics in theory and practice. (pp. 301-325). Dordrecht, Netherlands: Springer.

Anderson, C. B., Rozzi, R., Torres-Mura, J. C., McGehee, S. M., Sherriffs, M. F., Schüttler, E., \& Rosemond, A. D. (2006). Exotic vertebrate fauna in the remote and pristine Sub-An. Biodiversity and Conservation, 15, 3295-3313.

Arango, X., Rozzi, R., Massardo, F., Anderson, C. B., \& Ibarra, J. T. (2007). Descubrimiento e implementación del 
pájaro carpintero gigante (Campephilus magellanicus) como especie carismática: una aproximación biocultural para la conservación de la Reserva de Biosfera Cabo de Hornos. Magallania, 35(2), 71-88.

Armesto, J. J., Núñez-Ávila, M., Donoso, P., \& Rozzi, R. (2014). Introducción a la Sección Especial de Revista Bosque: Avances de una red de sitios de estudios ecológicos a largo plazo en el suroeste de Sudamérica. Bosque, 35, 413-414.

Armesto, J. J., Rozzi, R., Smith-Ramírez, C., \& Arroyo, M. T. K. (1998). Effective conservation targets in South American temperate forests. Science, 282, 12711272.

Bellard, C., Cassey, P., \& Blackburn, T. M. (2016). Alien species as a driver of recent extinctions. Biology Letters, 12, pp. doi: 10.1098/rsbl.2015.0623.

Berchez, F., Mansilla, A., Ghilardi-Lopes, N., P Schwindt, E., Leite, K., \& Rozzi, R. (2015). Ecology and Education in Marine Protected Areas: Insights from Brazil and South America. In R. Rozzi, F. S. Chapin III, J. B. Callicott, S. T. A. Pickett, M. E. Power, J. J. Armesto \& R. H. May Jr. (Eds.), Earth Stewardship: Linking Ecology and Ethics in Theory and Practice (pp. 351-366). Dordrecht, Netherlands: Springer.

Berghöfer, U., Rozzi, R., \& Jax, K. (2008). Local versus global knowledge: Diverse perspectives on nature in the Cape Horn biosphere reserve. Environmental Ethics, 30(3), 273-294.

Callicott, J. B. (1980). Animal Liberation: A triangular affair. Environmental Ethics, 2, 311-338.

Catford, J. A., Jansson, R., \& Nilsson, C. (2009). Reducing redundancy in invasion ecology by integrating hypotheses into a single theoretical framework. Diversity and Distributions, 15, 22-40.

Crego, R. D., Jiménez, J. E., Soto, C., Barroso, O., \& Rozzi, R. (2014). Tendencias poblacionales del visón norteamericano invasor (Neovison vison) y sus principales presas nativas desde su arribo a isla Navarino, Chile. Boletín de La Red Latinoamericana Para El Estudio de Especies Invasoras, 4, 4-18.

Crego, R. D., Jimenez, J. E., \& Rozzi, R. (2015). Expansión de la invasión del Visón Norteamericano (Neovison vison) en la Reserva de la Biosfera de Cabo de Hornos, Chile. Anales del Instituto de la Patagonia, 43, 157-162.

Crego, R. D., Jiménez, J. E., \& Rozzi, R. (2016). A synergistic trio of invasive mammals? Facilitative interactions among beavers, muskrats, and mink at the southern end of the Americas. Biological Invasions, 18, 19231938.
Crego, R. D., Jiménez, J. E., \& Rozzi, R. (2018a). Macroand micro-habitat selection of small rodents and their predation risk perception under a novel invasive predator at the southern end of the Americas. Mammal Research, 63, 1-9.

Crego R. D., Jiménez J. E., \& Rozzi R. (2018b). Potential niche expansion of the American mink invading a remote island free of native-predatory mammals. Plos One 13, p.e0194745

Crosby Jr, A. W. (1972). The Columbian exchange. Biological and cultural consequences of 1492. Westport, Connecticut, USA: Greenwood Press. INC.

Damasio, A. (1994). Descartes's error: emotion, reason and the human brain. New York, USA: G. P. Putnam's Sons.

Davis, E. F., Anderson, C. B., Valenzuela, A. E. J., Cabello, J. L., \& Soto, N. (2012). American mink (Neovison vison) trapping in the Cape Horn Biosphere Reserve: enhancing current trap systems to control an invasive predator. Acta Zoologica Fennica, 49, 18-22.

Ehrenfeld, J. G. (2010). Ecosystem consequences of biological invasions. Annual Review of Ecology, Evolution, and Systematics, 41, 59-80.

Essl, F., Bacher, S., Blackburn, T. M., Booy, O., Brundu, G., Brunel, S., .... Jeschke, J. M. (2015). Crossing frontiers in tackling pathways of biological invasions. BioScience, 65, 769-782.

Hargrove, E. C. (1989). Foundations of Environmental Ethics. Englewood Cliffs, New Jersey, USA: Prentice Hall.

Hutchins, M., \& Wemmer, C. (1986). Wildlife conservation and animal rights: Are they compatible? In M. W. Fox \& L. D. Mickley (Eds.), Advances in animal welfare science 1986/87 (Vol. 3, pp. 111-137). Washington, DC, USA: The Humane Society of the United States.

Ibarra, J. T., Fasola, L., Macdonald, D. W., Rozzi, R., \& Bonacic, C. (2009). Invasive American mink Mustela vison in wetlands of the Cape Horn Biosphere Reserve, southern Chile: what are they eating? Oryx, 43, 87-90.

Jaksic, F. M., Iriarte, J. A., Jiménez, J. E., \& Martínez, D. R. (2002). Invaders without frontiers: cross-border invasions of exotic mammals. Biological Invasions, 4, 157-173.

Jiménez, J. E., Crego, R. D., Soto, G. E., Román, I., Rozzi, R., \& Vergara, P. M. (2014). Potential impact of the alien American mink (Neovison vison) on Magellanic woodpeckers (Campephilus magellanicus) in Navarino Island, Southern Chile. Biological Invasions, 16, 961966. 
Larivière, S. (1999). Mustela vison. Mammalian Species, 608, 1-9.

Leopold, A. (1949). A Sand County Almanac. New York, USA: Oxford University Press.

Li, B., Parr, T., \& Rozzi, R. (2015) Geographical and Thematic Distribution of Publications Generated at the International Long-Term Ecological Research Network (ILTER) Sites. In R. Rozzi, F. S. Chapin III, J. B. Callicott, S. T. A. Pickett, M. E. Power, J. J. Armesto \& R. H. May Jr. (Eds.), Earth Stewardship: Linking Ecology and Ethics in Theory and Practice (pp. 351366). Dordrecht, Netherlands: Springer.

Liljesthröm, M., Schiavini, A., Sáenz Samaniego, R., Fasola, L., \& Raya Rey, A. (2013). Kelp Geese (Chloephaga hybrida) and Flightless Steamer-Ducks (Tachyeres pteneres) in the Beagle Channel: the importance of islands in providing nesting habitat. The Wilson Journal of Ornithology, 125, 583-591.

Llancabure, J. C. (2011). Relaciones entre el crecimiento de Nothofagus betuloides y el clima local y de gran escala en bosques subantárticos de la isla Navarino. Tesis de grado. Universidad Austral de Chile.

Lombardi, F., Cocozza, C., Lasserre, B., Tognetti, R., \& Marchetti, M. (2011). Dendrochronological assessment of the time since death of dead wood in an old growth Magellan's beech forest, Navarino Island (Chile). Austral Ecology, 36(3), 329-340.

McKinney, M. L., \& Lockwood, J. L. (1999). Biotic homogenization: A few winners replacing many losers in the next mass extinction. Trends in Ecology and Evolution, 14, 450-453.

Milton, K. (2002). Love of nature. Toward an ecology of emotions. New York, USA: Routledge.

Mooney, H. A., \& Cleland, E. E. (2001). The evolutionary impact of invasive species. PNAS, 98, 5446-5451.

Naess, A. (1973). The shallow and deep ecology movements. Inquiry, 16, 95-100.

Nelson, R. K. (1984). Make Prayers to the Raven. A Koyukon View of the Northern Forest. Chicago, USA: The University of Chicago Press.

O'Connell, A. F., Nichols, J. D., \& Karanth, K. U. (2011). Camera Traps in Animal Ecology. Methods and Analyses. Tokyo, Japan: Springer.

Palmer, C. (2013). Contested Frameworks in Environmental Ethics. In R. Rozzi, S. Pickett, C. Palmer, J. Armesto \& J. Callicott (Eds.), Linking Ecology and Ethics for a Changing World: Values, Philosophy, and Action (pp. 191-206). Dordrecht, Netherlands: Springer.

Regan, T. (1975). The moral basis of vegetarianism. Canadian
Journal of Philosophy, 5, 181-214.

Rozzi, R. (2004). Implicaciones éticas de Narrativas Yaganes y Mapuches sobre las aves de los bosques templados de Sudamérica austral. Ornitologia Neotropical, 15, 435-444.

Rozzi, R. (2012). Biocultural Ethics: Recovering the vital links between the inhabitants, their habits, and habitats. Environmental Ethics, 43, 27-50.

Rozzi, R. (2015a). Earth Stewardship and the Biocultural Ethic: Latin American Perspectives. In R. Rozzi, F. S. Chapin III, J. B. Callicott, S. T. A. Pickett, M. E. Power, J. J. Armesto \& R. H. May Jr. (Eds.), Earth Stewardship. Linking Ecology and Ethics in Theory and Practice. (pp. 87-112). Dordrecht, Netherlands: Springer.

Rozzi, R. (2015b). Implications of the Biocultural Ethic for Earth Stewardship. In R. Rozzi, F. S. Chapin III, J. B. Callicott, S. T. A. Pickett, M. E. Power, J. J. Armesto \& R. H. May Jr. (Eds.), Earth Stewardship. Linking Ecology and Ethics in Theory and Practice (pp. 113136). Dordrecht, Netherlands: Springer.

Rozzi, R., \& Sherriffs, M. F. (2003). El visón (Mustela vison Schreber, Carnivora: Mustelidae), un nuevo mamífero exótico para la Isla Navarino. Anales del Instituto de La Patagonia, 31, 97-104.

Rozzi, R., Massardo, F., Anderson, C. B., Heidinger, K., \& Silander, J. A. (2006). Ten principles for biocultural conservation at the southern tip of the Americas: the approach of the Omora Ethnobotanical Park. Ecology and Society, 11, 43-70.

Rozzi, R., Arango, X., Massardo, F., Anderson, C., Heidinger, K., \& Moses, K. (2008a). Field environmental philosophy and biocultural conservation: the Omora Ethnobotanical Park educational program. Environmental Ethics, 30, 325-336.

Rozzi, R., Armesto, J.J., \& Frodeman, R. (2008b). Integrating ecological sciences and environmental ethics into biocultural conservation in South American temperate sub-Antarctic ecosystems. Environmental Ethics, 30, 229-234.

Rozzi, R., Anderson, C. B., Pizarro, J. C., Massardo, F., Medina, Y., Mansilla, A. O., Kennedy, J. H., Ojeda, J., ... Kalin, M. T. (2010). Field environmental philosophy and biocultural conservation at the Omora Ethnobotanical Park: Methodological approaches to broaden the ways of ("S") in Long-Term Socio-Ecological Research (LTSER) Sites. Revista Chilena de Historia Natural, 83, 27-68.

Rozzi, R., Armesto, J. J., Gutiérrez, J. R., Massardo, F., Likens, G. E., Anderson, C. B., ... Arroyo, M. (2012). 
Integrating Ecology and Environmental Ethics: Earth Stewardship in the Southern End of the Americas. BioScience, 62(3), 226-236.

Rozzi, R., Massardo, F., Contador, T., Crego, R. D., Méndez, M., Rijal, R., Cavieres, L., \& Jimenez, J. E. (2014). Filosofía ambiental de campo: ecología y ética en las redes LTER-Chile e ILTER. Bosque, 23, 1-27.

SAG-FONDEMA. (2008). Programa control del visón en la provincia Antártica chilena. Punta Arenas, Chile.

Sarrazin, F., \& Lecomte, J. (2016) Evolution in the Anthropocene. Science, 351, 922-923.

Schüttler, E., Cárcamo, J., \& Rozzi, R. (2008). Diet of the American mink Mustela vison and its potential impact on the native fauna of Navarino Island, Cape Horn Biosphere Reserve, Chile. Revista Chilena de Historia Natural, 81, 585-598.

Schüttler, E., Klenke, R., McGehee, S., Rozzi, R., \& Jax, K. (2009). Vulnerability of ground-nesting waterbirds to predation by invasive American mink in the Cape Horn Biosphere Reserve, Chile. Biological Conservation, 142, 1450-1460.

Schüttler, E., Rozzi, R., \& Jax, K. (2011). Towards a societal discourse on invasive species management: A case study of public perceptions of mink and beavers in Cape Horn. Journal of Nature Conservation, 19, 175-184.

Simberloff, D. (2012). Nature, natives, nativism, and management: Worldviews underlying controversies in invasion biology. Environmental Ethics, 34, 5-25.

Simberloff, D. (2013). Introduced Species, Homogenizing Biotas and Cultures. In R. Rozzi, S. T. A. Pickett, C. Palmer, J. J. Armesto \& J. B. Callicott (Eds.), Linking Ecology and Ethics for a Changing World: Values,
Philosophy, and Action (pp. 33-48). Dordrecht, Netherlands: Springer.

Singer, P. (1975). Animal liberation. New York, USA: Random House.

Singer, P. (1993). Practical Ethics. Canadian Family Physician (Vol. 36). Cambridge, UK: Cambridge University Press.

Soga, M., \& Gaston, K. J. (2016). Extinction of experience: the loss of human-nature interactions. Frontiers in Ecology and the Environment, 14(2), 94-101.

Steadman, D. W. (2006). Extinction and biogeography of tropical Pacific birds. Chicago, USA: University of Chicago Press.

Steffen, W., Persson, Å., Deutsch, L., Zalasiewicz, J., Williams, M., Richardson, K., ... Svedin, U. (2011). The anthropocene: From global change to planetary stewardship. Ambio, 40, 739-761.

Valenzuela, A. E. J., Anderson, C. B., Fasola, L., \& Cabello, J. L. (2014). Linking invasive exotic vertebrates and their ecosystem impacts in Tierra del Fuego to test theory and determine action. Acta Oecologica, 54, 110-118.

Vilà, M., Espinar, J. L., Hejda, M., Hulme, P. E., Jarošík, V., Maron, J. L., ... Pyšek, P. (2011). Ecological impacts of invasive alien plants: a meta-analysis of their effects on species, communities and ecosystems. Ecology Letters, 14, 702-708.

Vitousek, P. P. M., D'Antonio, C. M. C., Loope, L. L., Rejmánek, M., \& Westbrooks, R. (1997). Introduced species: a significant component of human-caused global change. New Zealand Journal of Zoology, 21, 1-16.

Williamson, M. (1996). Biological Invasions. London, UK: Chapman \& Hall. 
